From the Association of Departments of Family Medicine

\section{PRESERVING PRIORITIES AMIDST A GLOBAL PANDEMIC: ADFM'S COMMITMENT TO HEALTH EQUITY}

This time last year, as our 2020 Annual Conference wrapped up in New Orleans, Louisiana, we had no clue how drastically the months ahead would change the course of the world at large; the COVID-19 pandemic brought forth radical change to institutions and systems. One of the largest changes for ADFM was the switch from our traditionally in-person Annual Conference to a virtual event. Despite its virtual nature, our speakers, panelists, and attendees cultivated robust conversations about COVID-19 impacts and steps to improve and continue best practices toward health equity.

Exploring the avenues of political and social determinants of health within the pandemic was this year's keynote speaker, Daniel Dawes, JD. Dawes is the director of the Satcher Health Leadership Institute at Morehouse School of Medicine in Atlanta, Georgia and a professor of health law, policy, and management. He expounded on ways to repair the political past that has created inequities in health care, currently exacerbated by the COVID-19 pandemic. He elaborated on the way both social and political constructs work in tandem to produce greater social inequities that must be addressed. Political and social determinants long predate COVID-19, so it is of no surprise that the impact of a pandemic (especially a highly politicized one) aggravated inequity. Dawes eloquently stated, "many [medical] issues can be firmly linked to political action or inaction. By understanding the political determinants of health, their origins, their impact, and their interconnection with the other determinants of health, we will be better equipped to develop and implement actionable solutions to close the health gap."

The speech concluded with actions to increase healthy equity: (1) engage in the tough conversations around race, place, and class; $(2)$ work upstream to understand the social and political determinants; $;$ (3) research the history of our communities to understand policies that exacerbated exclusion ${ }_{i}(4)$ strengthen networks and engagement with allies; and (5) realize that health equity both begins and ends with political determinants of health.
Many other panels focused on addressing health inequities, including:

- "Learning \& Unlearning: Addressing Structural Barriers to URM Faculty Achievement" led by STFM President, Tricia Elliot. The discussion on underrepresented minorities was presented by personal stories from the panel through an examination of how structural racism is at play across the medical education pipeline, including its impact on diverse representation in academic family medicine.

- The ADFM Education Transformation Committee led a panel titled "Moving the Needle on Racial Justice in Medical Education," with a strong focus on action plans to make notable steps toward being anti-racist. Recommendations of action plans highlighted were (1) implementing audacious statements from leadership;

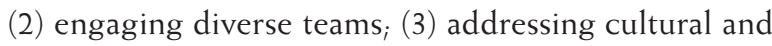
practical barriers; and (4) tracking progress.

- The ADFM Diversity, Health Equity, and Inclusion Committee led an interactive session on a 3-pillar framework for creating an anti-racism plan within your family medicine department. This framework highlighted concepts of diversity, equity, and inclusion within the departmental pillars of care delivery, health workforce recruitment \& retention, and learner recruitment \& training.

The theme of dedication and commitment to equity and diversity did not waver while also observing COVID-19; instead, COVID-19 became a highlighter of disparities. Rebecca Etz, PhD, Co-Director of the Larry A. Green Center, began the evaluation of COVID-19's impacts by presenting results of an ongoing survey of primary care providers and patients. The survey was built to be responsive to the pandemic practice and to date, has found major impacts on equitable primary care.

Among primary care providers:

- Nearly $60 \%$ report they have furloughed staff

- $25 \%$ of clinician salaries skipped

- $22 \%$ thinking of leaving primary care

- $48 \%$ burnout is at an all-time high

- $65 \%$ report family well-being suffers

- $45 \%$ decrease in psychological well-being

The survey also found that patients themselves have experienced major impacts.

- Inequities grew substantially: nearly $60 \%$ reported financial struggle, $40 \%$ substance abuse, $37 \%$ housing insecurity, 35\% food insecurity, 15\% domestic violence increase.

- A staggering $85 \%$ reported mental health decreased(ing), and physical health (58\%) and chronic conditions worsening (38\%) was also reported.

So, where do we go from here? An important and valuable reminder in the survey is that patients 
reported highly valuing the relationship with their care provider. Primary care must continue to pivot in the face of adversity and maintain the relationship - $a$ message that was both validating and re-energizing for conference attendees.

The conference wrapped with 2 final, stellar sessions. First, "A Look into the Life of a Dean," shared by a panel of Deans who were formerly chair of Family Medicine departments. And a final note on a systemsapproach to well-being, highlighting specific examples of methods to care for one's self and one's team during the chaotic time in which we find ourselves. This year's 2021 conference bore the weight of a pandemic and exacerbated racial, economic, and political divisions and disparity. In spite of all the turmoil, the focus on well-being and the positive impacts of family medicine revealed that this passion managed to bring us together while we sat hundreds of miles apart. This year was one for the records; now we look onward towards vaccinations, improving our systems, our practices, and aim to connect next year in Denver, Colorado.

Jessie Vera, ADFM Program and Communication Coordinator

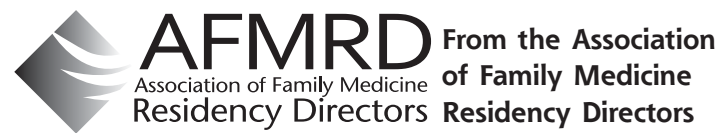

Ann Fam Med 2021;19:280-281. https://doi.org/10.1370/afm.2704.

\section{UNDERSTANDING AND USING THE ACGME RESIDENT SURVEYS TO IMPROVE YOUR RESIDENCY PROGRAM}

Since 2004, the Accreditation Council for Graduate Medical Education (ACGME) has administered an annual survey of all residents and fellows to monitor ongoing program quality and to serve as a possible indicator of the need to assess programs between site visits. With the introduction of the Next Accreditation System (NAS) ${ }^{1}$ in 2013, the annual survey took on even greater significance as the frequency of scheduled program site visits lengthened to every 10 years. While specific questions are only visible to the survey participants, general content areas include clinical experience/education, faculty teaching and supervision, evaluation, educational content, diversity and inclusion, program resources, patient safety and teamwork, professionalism, and overall satisfaction. ${ }^{2}$ In family medicine, additional questions are directed at continuity, care across settings, and family-centered care.

Release of the secure survey link typically occurs in February of each year with completion required in a 5 -week window by a minimum of $70 \%$ of residents in order to see the aggregate results. An exception has occurred in the 2021 survey as the result of the pandemic, permitting 8 weeks for response. The program is responsible for distribution of the secure link to all participants and for ensuring completion, as the ACGME does not remind residents. Programs are prohibited from directing residents in their responses. The survey is considered complete when all questions are answered and the resident selects the "submit" button. Program results aggregated by domain are subsequently published as a summary in the ACGME ADS system with national specialty comparison data. At no time are individual respondents identified, nor are the questions made available to the program director, faculty, or program coordinator. ${ }^{3}$

Historically, concerns about differing interpretations of questions have been reported, as well as inconsistency in the directionality of the frequency norms used. ${ }^{4}$ Specific areas of concern include the balance between service and education, in-house call vs night float, and competition from other learners. ${ }^{5}$ So how should program directors balance the importance of resident autonomy and confidentiality with maximizing accuracy in their responses? ${ }^{6}$ Because of the high-stakes nature of the survey, program directors should ensure residents understand the questions and their context.

Programs can consider offering an internal survey well in advance of the ACGME survey to identify areas that may need clarification. Many interns taking the survey may be unaware of specific resources or may not recall items discussed during orientation. Programs often conduct a residency meeting that includes faculty to review program resources and policies, especially those related to the clinical learning environment, duty hours, diversity and inclusion, and resident wellness. Discussing these resources and policies can help remind residents of the opportunities within the program and the institution to express and resolve concerns. ${ }^{7}$ Programs should also review the response options for questions related to frequency and occurrences to reinforce a shared understanding of their definitions. ${ }^{8}$

Aggregate results are normally provided to program directors in May or June. It is expected that these results be reviewed as part of the Annual Program Evaluation, addressing any areas that are "negative outliers" compared to national averages. Also, programs should review the trends over the past few years to determine if they are positive or going in a downward direction. This is a time to identify areas of improvement that can be part of the next academic year's strategic plan. ${ }^{9}$ Be sure to address these areas in 